\title{
Synthesis of novel four-fused-ring chromeno-benzoxepinones from salicylaldehydes and 1-benzoxepin-5-ones via the oxa-Michael reaction/aldol condensation
}

\author{
Po-Yuan Chen, ${ }^{\text {C Chia-Ying Zhon, }{ }^{\text {a }} \text { Hsing-Ming Chen,b Chih-Hui Yang, }}{ }^{\mathrm{c}}$ \\ Tzu-Pin Wang,*,a and Eng-Chi Wang*,a \\ ${ }^{a}$ Department of Medicinal and Applied Chemistry, Kaohsiung Medical University, \\ Kaohsiung 807, Taiwan \\ ${ }^{b}$ Department of Nutrition and Health Science, Fooyin University, Kaohsiung 831, Taiwan \\ ${ }^{c}$ Department of Biological Science \& Technology, I-Shou University, Kaohsiung, Taiwan \\ E-mail: enchwa@kmu.edu.tw
}

\begin{abstract}
In this study, the synthesis of a series of novel four-fused-ring chromeno-benzoxepinones is described. The reaction of 1-benzoxepin-5-ones with salicylaldehydes mediated by 1,4diazabicyclo[2,2,2] octane (DABCO) involves domino reactions including an oxa-Michael reaction, an aldol condensation and dehydration in a one-pot sequence to yield the target compounds in fairly good yields.
\end{abstract}

Keywords: Salicylaldehydes, oxa-Michael/aldol condensation, 1-benzoxepin-5-ones, DABCO, fur-fused ring compounds

\section{Introduction}

The oxa-Michael reaction has become a useful, concise and efficient tool for the synthesis of oxycyclic compounds, and was recently reviewed by Nising et al. ${ }^{1}$ Furthermore, the reactions of salicylaldehydes with $\alpha, \beta$-unsaturated cyclic carbonyl compounds catalyzed by base to undergo the domino oxa-Michael and aldol condensation to provide an easy access to a variety of chromene derivatives have also been documented in the literature. For instance, the reaction of salicylaldehydes with $\alpha, \beta$-unsaturated cyclohexenone yielded three-fused ring compounds with 1-chromene fused to a cyclohexanone ring, providing the key intermediates for various bioactive tetrahydroxanthones, ${ }^{2}$ the reaction of salicylaldehydes with chiral substituted $\alpha, \beta$-unsaturated cyclohexenone via the Morita-Baylis-Hillman reaction or oxa-Michael addition generated the bioactive diversonol, ${ }^{3}$ the reaction of salicylaldehydes with $\alpha, \beta$-unsaturated aldehydes catalyzed by prolinol yielded chiral chromenes, ${ }^{4}$ the reaction of 2-(2-nitrovinyl)phenol with $\alpha, \beta$ - 
unsaturated aldehydes gave tetrahydro- $6 H$-benzo $[c]$ chromenes, ${ }^{5}$ the reaction of salicylaldehyde with $\alpha, \beta$-unsaturated aldehydes catalyzed by chiral amine/chiral acid afforded chiral chromenes, ${ }^{6}$ as well as others. ${ }^{7}$ Although numerous syntheses based on these oxa-Michael addition/aldol condensation have been well developed, the construction of four-fused-ring systems had not been studied. Therefore, to synthesize such four-fused-ring chromene derivatives is an attractive and important topic, not only for synthetic purposes, but also in the search for bioactive compounds. In continuing our studies on benzoheterocyclic compounds, ${ }^{8}$ herein we report the synthesis of a series of novel four-ring compounds, specifically 5a,6-dihydro- $12 \mathrm{H}$-chromeno[2,3-c][1]benzoxepin-12-ones, comprising a 1-benzoxepin-5-one and chromene units.

\section{Results and Discussion}

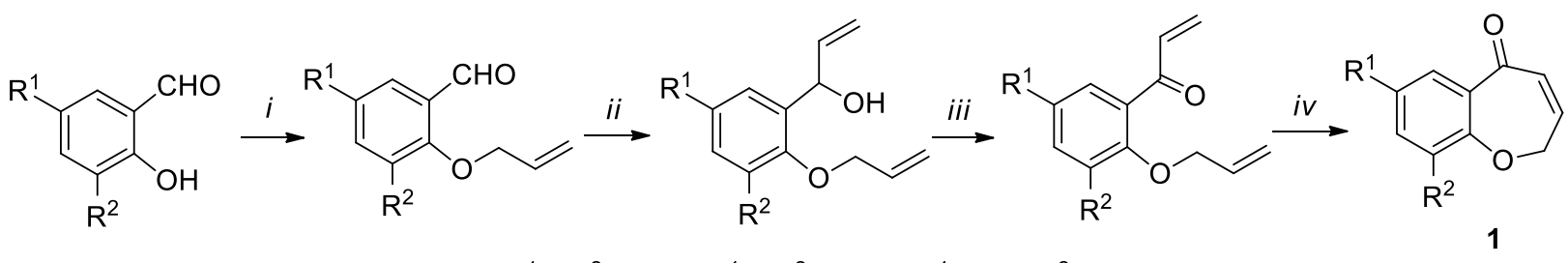

a. $R^{1}=R^{2}=H ; b \cdot R^{1}=R^{2}=C l ; c \cdot R^{1}=B r, R^{2}=H$

Reagents and conditions: $i$. allyl bromide, $\mathrm{K}_{2} \mathrm{CO}_{3}$, in refluxing $\mathrm{CH}_{3} \mathrm{CN}, 95-97 \%$; ii. vinyl magnesium bromide, in THF, 73-81\%; iii. $\mathrm{MnO}_{2}$ in $\mathrm{CH}_{2} \mathrm{Cl}_{2}, 71-80 \%$; iv. Grubbs catalyst (2 ${ }^{\text {nd }}$ generation), in $\mathrm{CH}_{2} \mathrm{Cl}_{2}, 81-84 \%$

Scheme 1. Preparation of the key intermediate benzoxepin-5-ones (1a-c) from salicylaldehydes. ${ }^{9}$

Our synthetic strategy is based on the reaction of the key intermediate 1-benzoxepin-5-ones (prepared from salicylaldehydes in four synthetic steps as outlined in Scheme $1^{9}$ ), with further salicylaldehydes, mediated by 1,4-diazabicyclo[2,2,2]octane (DABCO) (Scheme 2). Our synthetic strategy successfully provides a concise one-pot reaction to afford the target compounds in fairly good yields.

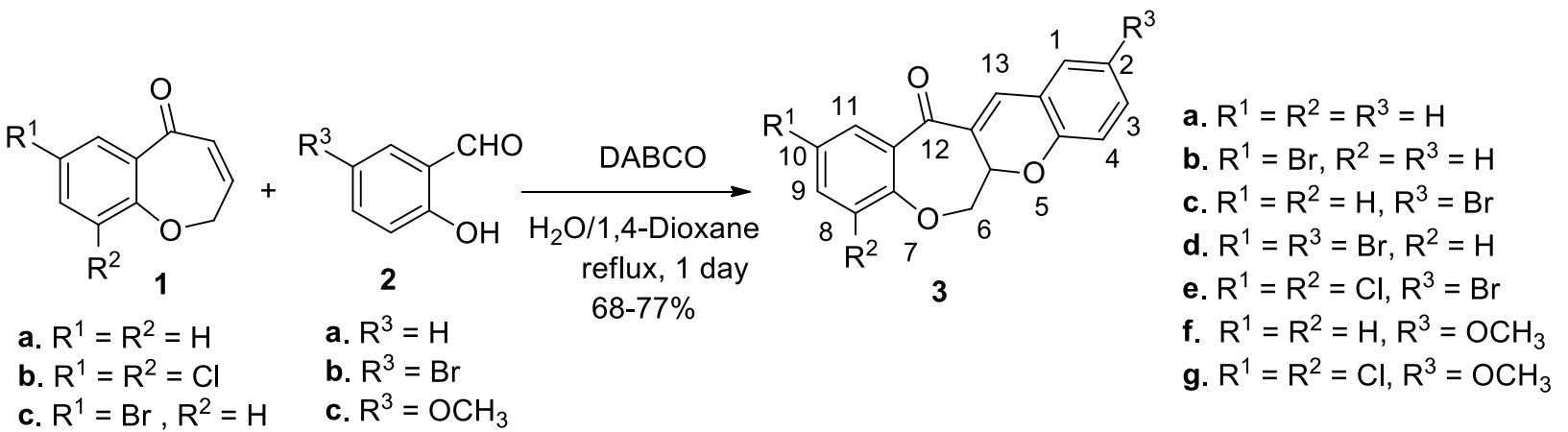


<smiles>[R]c1cc([R])c2c(c1)C(=O)C=C[C+]2</smiles><smiles>O=Cc1cc(Br)ccc1O</smiles>
a. $R^{1}=R^{2}=H$
b. $\mathrm{R}^{1}=\mathrm{R}^{2}=\mathrm{Cl}$
c. $\mathrm{R}^{1}=\mathrm{Br}, \mathrm{R}^{2}=\mathrm{H}$

a. $\mathrm{R}^{3}=\mathrm{H}$

b. $\mathrm{R}^{3}=\mathrm{Br}$

c. $\mathrm{R}^{3}=\mathrm{OCH}_{3}$

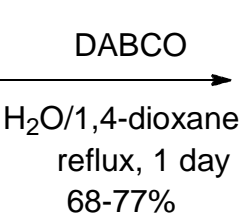

$68-77 \%$

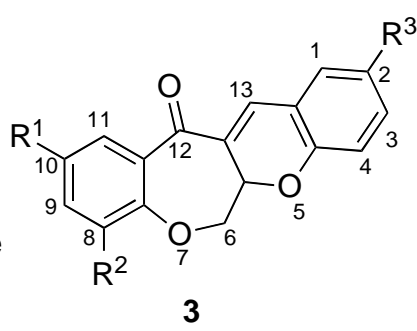

3 a. $R^{1}=R^{2}=R^{3}=H$

b. $R^{1}=B r, R^{2}=R^{3}=H$

c. $R^{1}=R^{2}=H, R^{3}=B r$

d. $R^{1}=R^{3}=B r, R^{2}=H$

e. $R^{1}=R^{2}=C l, R^{3}=B r$

f. $R^{1}=R^{2}=H, R^{3}=O_{3}$

g. $\mathrm{R}^{1}=\mathrm{R}^{2}=\mathrm{Cl}, \mathrm{R}^{3}=\mathrm{OCH}_{3}$

Scheme 2 The reaction of 1-benzoxepin-5-ones (1) with salicylaldehydes (2) mediated by DABCO to yield the four-ring compounds, chromenobenzoxepinones 3.

Initially, 1-benzoxepin-5-one (1a) was allowed to react with 5-bromosalicylaldehyde (2b) as a model reaction. Various solvents and two tertiary amines were investigated to determine appropriate conditions for obtaining the target compound, 2-bromo-5a,6-dihydro-12 $\mathrm{H}$ chromeno[2,3-c][1]benzoxepin-12-one (3c). The results obtained are presented in Table 1.

Table 1. Comparison of various conditions ${ }^{\mathrm{a}}$ for the synthesis of $\mathbf{3 c}$ from $\mathbf{1 a}$ and $\mathbf{2 b}$<smiles>O=C1C=CCOc2ccccc21</smiles>

$1 \mathrm{a}$<smiles>O=C1C=CCOc2ccccc21</smiles>

$1 a$<smiles>O=Cc1cc(Br)ccc1O</smiles>

2b<smiles>O=Cc1cc(Br)ccc1O</smiles>

$2 b$

\section{Conditions}

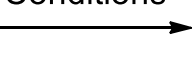

conditions<smiles>O=C1C2=Cc3cc(Br)ccc3OC2COc2ccccc21</smiles>

$3 c$

\begin{tabular}{cccc}
\hline Entry & Solvent & Tertiary amine & Yield $(\%)^{\mathrm{b}}$ \\
\hline 1 & $\mathrm{H}_{2} \mathrm{O}$ & DABCO & $28^{\mathrm{c}}$ \\
2 & $1,4-$ dioxane & DABCO & $49^{\mathrm{c}}$ \\
3 & $\mathrm{CH}_{3} \mathrm{OH}$ & DABCO & $42^{\mathrm{c}}$ \\
4 & $\mathrm{CH}_{2} \mathrm{Cl}_{2}$ & DABCO & $15^{\mathrm{c}}$ \\
5 & $\mathrm{DMSO}$ & DABCO & $55^{\mathrm{c}}$ \\
6 & $\mathrm{H}_{2} \mathrm{O} / \mathrm{DMSO}(2: 1)$ & DABCO & 60 \\
7 & $\mathrm{H}_{2} \mathrm{O} / 1,4-$ dioxane $(2: 1)$ & DABCO & 73 \\
\hline
\end{tabular}


Table 1. Continued

\begin{tabular}{cccc}
\hline Entry & Solvent & Tertiary amine & Yield (\%)b \\
\hline 8 & $\mathrm{H}_{2} \mathrm{O} / 1,4$-dioxane $(2: 1)$ & $\mathrm{DBU}^{\mathrm{d}}$ & $46^{\mathrm{c}}$ \\
\hline
\end{tabular}

${ }^{a}$ General procedure: A mixture of 5-bromosalicylaldehyde (2b) (5 mmol) and 1-benzoxepin-5one (1a) $(5 \mathrm{mmol})$ in solvent $(60 \mathrm{~mL})$ was mixed with amine $(11 \mathrm{mmol})$. The resulting mixture was stirred at reflux for 1 day. ${ }^{b}$ Isolated from column chromatography. ${ }^{c}$ Accompanied with recovery of starting material. ${ }^{\mathrm{d}} \mathrm{DABCO}=1,4$-diazabicyclo[2,2,2] octane ; DBU $=1,8$ diazabicyclo[5.4.0]undec-7-ene.

As shown in Table 1, in 1,4-dioxane: $\mathrm{H}_{2} \mathrm{O}=1: 2$ as reaction solvent, DABCO as base, and at the reflux temperature $\left(100-102{ }^{\circ} \mathrm{C}\right)$, 3c was produced in the highest yield (73\%, entry 7 ). Comparing those reaction conditions, we found the utilization of $\mathrm{H}_{2} \mathrm{O}$ as a sole reaction solvent without any organic solvent and the utilization of DABCO as a reaction base gave a lower yield (28\%) (entry 1). Furthermore, other organic solvents such as 1,4-dioxane, $\mathrm{CH}_{3} \mathrm{OH}$, and DMSO used as reaction solvent, and DABCO used as base, did not improve the yields (42-55\%) (entries 2, 3 and 5). When $\mathrm{CH}_{2} \mathrm{Cl}_{2}$ was used as reaction solvent and DABCO as base, the yield of 3c was lower $(15 \%)$ than other conditions and starting materials were recovered (entry 4 ). In order to improve the percentage yields, binary solvent systems were examined. In the mixed solvent system $\mathrm{H}_{2} \mathrm{O} / \mathrm{DMSO}(2: 1)$ and base DABCO, the reaction of $\mathbf{1 a}$ with $\mathbf{2 b}$ under reflux gave $\mathbf{3 c}$ in $60 \%$ yield (entry 6 ). Under the same reaction condition as entry 7 (dioxane: $\mathrm{H}_{2} \mathrm{O}=1: 2$, DABCO, and at reflux) but DABCO was replaced by DBU as a reaction base, the yield of compound 3c was $46 \%$ (entry 8). Finally, the reaction of $\mathbf{1 a}$ with $\mathbf{2 b}$ in the mixed solvents $1,4-$ dioxane $/ \mathrm{H}_{2} \mathrm{O}$ $(1: 2)$ and the use of DABCO base under reflux gave $\mathbf{3 c}$ in $73 \%$ yield (entry 7 ), the highest yield. Thus, the reaction trend of solvent systems we found in this reaction is $\mathrm{H}_{2} \mathrm{O} / 1$,4-dioxane > $\mathrm{H}_{2} \mathrm{O} / \mathrm{DMSO}>\mathrm{DMSO}>1$,4-dioxane $>\mathrm{CH}_{3} \mathrm{OH}>\mathrm{H}_{2} \mathrm{O}>\mathrm{CH}_{2} \mathrm{Cl}_{2}$. For the tertiary amine used in the reaction, we found that $\mathrm{DABCO}$ is superior to $\mathrm{DBU}$ in giving the final product.

In our synthetic strategy, two possible reaction pathways may occur to yield the same target products. One pathway could go initially through the oxa-Michael reaction and the other one may start with a Baylis-Hillman reaction. In order to understand which reaction pathway is more possible, 1-benzoxepin-5-one (1a) was reacted with o-benzyloxybenzaldehyde in which the phenolic $\mathrm{OH}$ is protected, under the conditions which gave a high yield, described above i.e., solvent, $\mathrm{H}_{2} \mathrm{O} / 1$,4-dioxane $=2: 1$; base, DABCO, at reflux. After $24 \mathrm{~h}$, we found that no BaylisHillman adduct was obtained, just the recovery of both starting materials (Scheme 3 ). This result strongly suggests that the reaction of 1-benzoxepin-5-one (1a) with $o$-hydroxybenzaldehyde mediated by DABCO may predominantly be via a domino sequence of reactions involving an oxa-Michael reaction, aldol condensation, and dehydration. 
<smiles>O=C1C=CCOc2ccccc21</smiles>

1-benzoxepin-5-one (1a)<smiles>O=C1C=CCOc2ccccc21</smiles>

1-benzoxepin-5-one (1a)<smiles>O=Cc1ccccc1OCc1ccccc1</smiles><smiles>[H][R18]([H])([2H])C(C)C</smiles>
o-Benzyloxybenzaldehyde

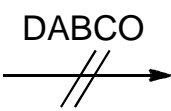
o-benzyloxybenzaldehyde<smiles>O=C1C(C(O)c2ccccc2Br)=CCOc2ccccc21</smiles>

The Baylis-Hillman Adduct<smiles>O=C1C(C(O)c2ccccc2OBr)=CCOc2ccccc21</smiles>

the Baylis-Hillman adduct

Scheme 3. The reaction of 1-benzoxepin-5-one (1a) with $o$-benzyloxybenzaldehyde mediated by DABCO in $\mathrm{H}_{2} \mathrm{O} /$ 1,4-dioxane (2:1) at reflux.

Therefore, the formation of $\mathbf{3} \mathbf{a}$ from the reaction of $\mathbf{1 a}$ and $\mathbf{2} \mathbf{a}$, as an example, could be rationally illustrated by the mechanism shown in Scheme 4. Initially, the phenolic proton of salicylaldehyde (2a) is abstracted by DABCO to generate a phenolate anion (step 1). The anion then attacks 1-benzoxepin-5-one (1a) in a Michael addition and yields the intermediate I (step 2), which then undergoes an intramolecular crossed aldol reaction to afford the intermediate II (step 3). This then picks up a proton from protonated DABCO to generate the intermediate III and regenerate free DABCO (step 4). Finally, the intermediate III is dehydrated by initial deprotonation by DABCO and hydroxide ion elimination to yield the final product, compound 3a (step 5).

According to the best reaction conditions we found, the reactions of 1a-c with 2a-c were carried out mediated by DABCO in 1,4-dioxane: $\mathrm{H}_{2} \mathrm{O}=1: 2$ at reflux, to afford compounds 3a-g in $68-77 \%$ yields. 
1)

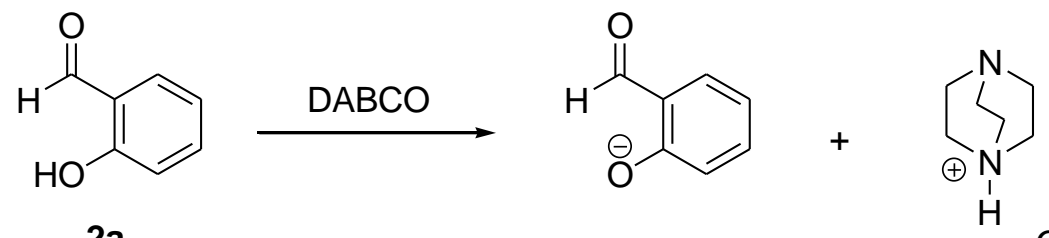

2)

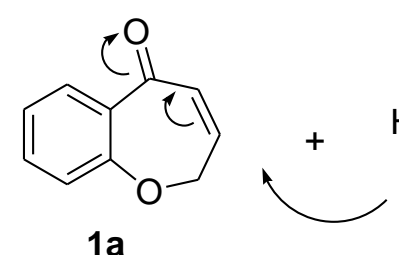<smiles>O=Cc1ccccc1[O-]</smiles><smiles>O=Cc1ccccc1OC1C=C(O)c2ccccc2OC1</smiles>

3)<smiles>O=C(O)C1=CC2(COc3ccccc31)COc1ccccc1C2CO</smiles><smiles>O=C1c2ccccc2OCC2Oc3ccccc3C(O)C12</smiles>

4)<smiles>O=C1c2ccccc2OCC2Oc3ccccc3C(O)C12</smiles>

II

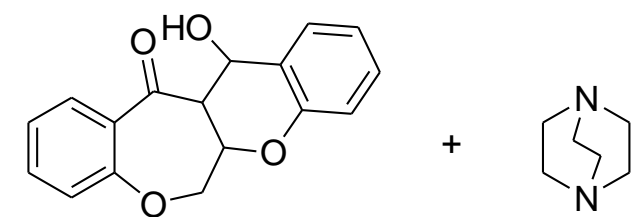

III

5)<smiles>O=C1c2ccccc2OCC2Oc3ccccc3C(O)C12</smiles>

III

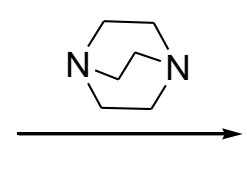<smiles>O=C1C2=Cc3ccccc3OC2COc2ccccc21</smiles>

$3 a$

Scheme 4. The proposed mechanism of formation of $\mathbf{3 a}$ from the reaction of $\mathbf{1 a}$ and $\mathbf{2 a}$.

The structural elucidation of compounds 3a-g is mainly based on their ${ }^{1} \mathrm{H}-\mathrm{NMR}$ spectra, ${ }^{13} \mathrm{C}$ NMR, EI-MS and HRMS or EA. For instance, the ${ }^{1} \mathrm{H}-\mathrm{NMR}$ spectrum of 2,10-dibromo-5a,6dihydro-12H-chromeno[2,3-c][1]benzoxepin-12-one (3d) was assigned as follows. Two double doublet $\mathrm{sp}^{3}$-protons, one at $\delta 4.38$ and the other at $\delta 4.79$, indicate the signals of H-6a and H-6b which are coupled to each other and commonly coupled with $\mathrm{H}-5 \mathrm{a}$, respectively. The signal at $\delta$ 5.14 with multiplet splitting (ddd), coupled with $\mathrm{H}-13$, H-6a and H-6b, was assigned to H-5a. An aromatic proton with a doublet $(J 8.8 \mathrm{~Hz})$ signal at $\delta 6.86$ was assigned to $\mathrm{H}-4$. The proton with a doublet signal at $\delta 7.02$, which has a typical ortho coupling constant $(J 8.8 \mathrm{~Hz}$ ), was assigned to $\mathrm{H}-8$. The doublet signal at $\delta 7.28$ that has an allylic coupling $(J 1.6 \mathrm{~Hz})$ with $\mathrm{H}-5 \mathrm{a}$, was assigned to $\mathrm{H}-13$. The proton with a doublet $(\mathrm{J} 2.4 \mathrm{~Hz})$ signal at $\delta 7.36$ was assigned to $\mathrm{H}-1$, the proton 
with a double doublet $(J 9.2,2.4 \mathrm{~Hz})$ signal at $\delta 7.38$ was assigned to $\mathrm{H}-3$, The correlation of $\mathrm{H}-1$ linked with H-3, and H-3 linked with $\mathrm{H}-4$ was also proved by the COSY technique. The proton with a double doublet signal at $\delta 7.56$ which has a typical ortho and meta coupling constant $J$ $8.8,2.8 \mathrm{~Hz}$ was assigned to H-9. Similarly, the proton with a doublet signal at $\delta 8.24$ which has a meta coupling constant $J 2.8 \mathrm{~Hz}$ was assigned to $\mathrm{H}-11$. The other spectral data of compound $\mathbf{3 d}$ such as ${ }^{13} \mathrm{C}-\mathrm{NMR}$, EI-MS and HRMS are all consistent with the proposed structure.

For further structural confirmation, an X-ray analysis of 2,10-dibromo-5a,6-dihydro- $12 \mathrm{H}$ chromeno[2,3-c][1]benzoxepin-12-one (3d) was undertaken. The resulting ORTEP, which is consistent with the structure of compound 3d, is depicted in Figure 1.

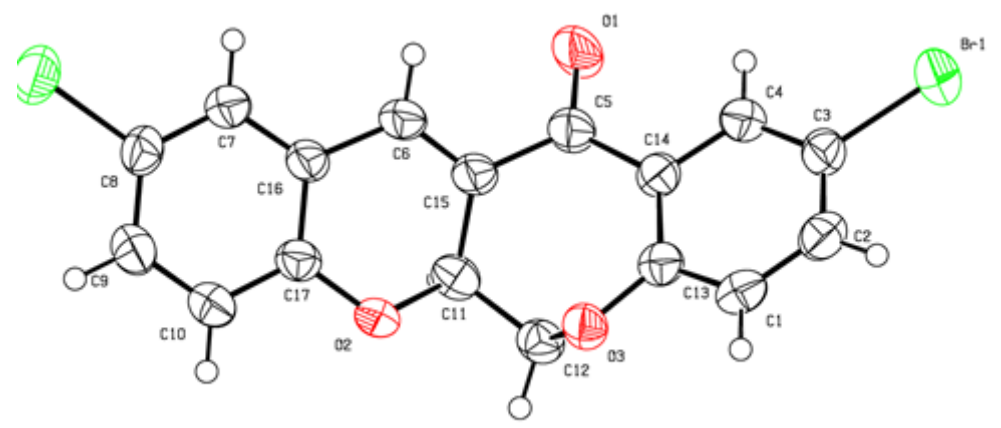

Figure 1. The ORTEP diagram of 2,10-dibromo-5a,6-dihydro-12H-chromeno[2,3-c][1]benzoxepin-12-one [or 3,8-dibromo-11a,12-dihydro-11,13-dioxabenzo[4,5]cyclohepta[1,2-b]naphthalen-5-one] (3d). ${ }^{10}$

All structures of these novel four-fused ring benzooxycyclic compounds 3a-g obtained from the oxa-Michael reaction/aldol condensation of $\mathbf{1 a - c}$ and $\mathbf{2 a - c}$, were confirmed by physical and spectral data, and HRMS. Their physical and selected spectral data were compiled in Table 2. 
Table 2. The physical and selected spectral data of compounds 3a-g

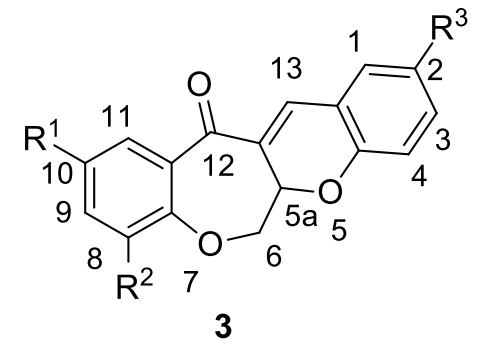

a. $\mathrm{R}^{1}=\mathrm{R}^{2}=\mathrm{R}^{3}=\mathrm{H}$

b. $R^{1}=B r, R^{2}=R^{3}=H$

c. $R^{1}=R^{2}=H, R^{3}=B r$

d. $R^{1}=R^{3}=B r, R^{2}=H$

e. $R^{1}=R^{2}=\mathrm{Cl}, R^{3}=\mathrm{Br}$

f. $R^{1}=R^{2}=H, R^{3}=\mathrm{OCH}_{3}$

g. $\mathrm{R}^{1}=\mathrm{R}^{2}=\mathrm{Cl}, \mathrm{R}^{3}=\mathrm{OCH}_{3}$

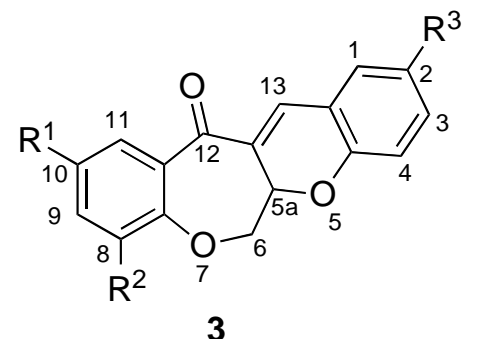
a. $R^{1}=R^{2}=R^{3}=H$
b. $R^{1}=B r, R^{2}=R^{3}=H$
c. $R^{1}=R^{2}=H, R^{3}=B r$
d. $R^{1}=R^{3}=B r, R^{2}=H$
e. $R^{1}=R^{2}=C l, R^{3}=B r$
f. $R^{1}=R^{2}=H, R^{3}=\mathrm{OCH}_{3}$
g. $\mathrm{R}^{1}=\mathrm{R}^{2}=\mathrm{Cl}, \mathrm{R}^{3}=\mathrm{OCH}_{3}$

\begin{tabular}{|c|c|c|c|c|c|c|c|}
\hline Compd & $\begin{array}{l}\text { Yield }^{a} \\
(\%)\end{array}$ & $\begin{array}{l}\text { IR } \\
\left(\mathrm{cm}^{-1}\right) \\
(\mathrm{C}=\mathrm{O})\end{array}$ & $\begin{array}{l}\mathrm{H}-13^{b} \\
\mathrm{~d}(J)\end{array}$ & $\begin{array}{l}\mathrm{H}-5 \mathrm{a}^{b} \\
\mathrm{ddd}(\mathcal{J})\end{array}$ & $\begin{array}{l}\mathrm{H}-6 \mathrm{a}^{b} \\
\mathrm{dd}(\mathrm{J})\end{array}$ & $\begin{array}{l}\mathrm{H}-6 \mathrm{~b}^{b} \\
\mathrm{dd}(J)\end{array}$ & $\begin{array}{cc} & \text { HRMS } \\
& \text { Formula } \\
\text { Calcd } & \text { Found } \\
\end{array}$ \\
\hline \multirow{2}{*}{$3 a$} & \multirow{2}{*}{68} & \multirow{2}{*}{1643} & 7.37 & 5.13 & 4.39 & 4.79 & $\mathrm{C}_{17} \mathrm{H}_{12} \mathrm{O}_{3}$ \\
\hline & & & (2.0) & $(4.0,2.8,2.0)$ & $(12.8,2.8)$ & $(12.8,4.0)$ & $264.0786 \quad 264.0788$ \\
\hline \multirow{2}{*}{$3 b$} & \multirow{2}{*}{72} & \multirow{2}{*}{1644} & 7.40 & 5.14 & 4.39 & 4.79 & $\mathrm{C}_{17} \mathrm{H}_{11} \mathrm{BrO}_{3}$ \\
\hline & & & (2.0) & $(4.0,2.8,2.0)$ & $(12.8,2.8)$ & $(12.8,4.0)$ & $341.9892 \quad 341.9889$ \\
\hline \multirow{2}{*}{$3 c$} & \multirow{2}{*}{73} & \multirow{2}{*}{1639} & 7.47 & 5.14 & 4.38 & 4.79 & $\mathrm{C}_{17} \mathrm{H}_{11} \mathrm{BrO}_{3}$ \\
\hline & & & (2.0) & $(4.0,2.8,2.0)$ & $(12.8,2.8)$ & $(12.8,4.0)$ & $341.9892 \quad 341.9895$ \\
\hline \multirow{2}{*}{$3 d$} & \multirow{2}{*}{75} & \multirow{2}{*}{1649} & 7.28 & 5.14 & 4.38 & 4.79 & $\mathrm{C}_{17} \mathrm{H}_{10} \mathrm{Br}_{2} \mathrm{O}_{3}$ \\
\hline & & & (2.0) & $(4.0,2.4,2.0)$ & $(13.2,2.4)$ & $(13.2,4.0)$ & $419.8997 \quad 419.8993$ \\
\hline \multirow{2}{*}{$3 e$} & \multirow{2}{*}{74} & \multirow{2}{*}{1644} & 7.46 & 5.18 & 4.42 & 4.85 & $\mathrm{C}_{17} \mathrm{H}_{9} \mathrm{BrCl}_{2} \mathrm{O}_{3}$ \\
\hline & & & (1.6) & $(4.8,3.2,1.6)$ & $(12.4,3.2)$ & $(12.4,4.8)$ & $409.9112 \quad 409.9113$ \\
\hline \multirow{2}{*}{$3 f$} & \multirow{2}{*}{77} & \multirow{2}{*}{1646} & 7.34 & 5.08 & 4.38 & 4.79 & $\mathrm{C}_{18} \mathrm{H}_{14} \mathrm{O}_{4}$ \\
\hline & & & (2.0) & $(4.0,2.8,2.0)$ & $(12.4,2.8)$ & $(12.4,4.0)$ & $294.0892 \quad 294.0893$ \\
\hline \multirow{2}{*}{$3 g$} & \multirow{2}{*}{76} & \multirow{2}{*}{1640} & 7.42 & 5.11 & 4.40 & 4.85 & $\mathrm{C}_{18} \mathrm{H}_{12} \mathrm{Cl}_{2} \mathrm{O}_{4}$ \\
\hline & & & $(1.6)$ & $(4.8,3.2,1.6)$ & $(12.4,3.2)$ & $(12.4,4.8)$ & $362.0113 \quad 362.0110$ \\
\hline
\end{tabular}

${ }^{a}$ Isolated yield from column chromatography; ${ }^{b}$ Measured using $400 \mathrm{MHz}{ }^{1} \mathrm{H}-\mathrm{NMR}$ in $\mathrm{CDCl}_{3}$

\section{Conclusions}

In this study we have established an efficient synthesis of various substituted 5a,6-dihydro- $12 \mathrm{H}$ chromeno[2,3-c][1]benzoxepin-12-ones, novel four-fused-ring heterocyclic compounds, via 
sequential reactions including an oxa-Michael addition/aldol condensation/dehydration in one pot. This study also demonstrated that in this reaction participation of the Baylis-Hillman reaction can be excluded. All title compounds have been fully characterized by spectral data such as ${ }^{1} \mathrm{H}-\mathrm{NMR},{ }^{13} \mathrm{C}-\mathrm{NMR}$ and HRMS. The structure of compound $\mathbf{3 d}$ was further confirmed by $\mathrm{X}$-ray diffraction.

\section{Experimental Section}

General. Melting points were measured with Yanaco micro melting-point apparatus. ${ }^{1} \mathrm{H}-\mathrm{NMR}$ and ${ }^{13} \mathrm{C}-\mathrm{NMR}$ spectra were obtained on a Varian Unity Plus 400 spectrometer. Chemical shifts are indicated in parts per million with respect to TMS. IR spectra were measured on a Perkin Elmer system 2000 FT-IR spectrometer. Elemental analyses were recorded on a Heraeus CHN-O Rapid analyzer. Mass spectra were recorded on a Chem/hp/middle spectrometer connected to a Hewlett Packard series II model gas-liquid chromatography. HRMS spectra were performed on a JEOL JMS SX/SX 102A instrument. Silica gel (230-400 mesh) for column chromatography and precoated silica gel plates (60 F-254) for TLC was purchased from E. Merck Co. UV light (254 $\mathrm{nm}$ ) was used to detect spots on TLC plates after development.

Salicylaldehydes (1a-c) were purchased from Aldrich Chemical Co. 2-Allyloxybenzaldehyde (97\%), 2-allyloxy-3,5-dichlorobenzaldehyde (95\%), 2-allyloxy-5-bromobenzaldehyde (96\%); 1(2-allyloxyphenyl)-2-propen-1-ol (81\%), 1-(2-allyloxy-3,5-dichlorophenyl)prop-2-en-1-ol (75\%), 1-(2-allyloxy-5-bromophenyl)- prop-2-en-1-ol (73\%); 1-(2-allyloxyphenyl)-2-propen-1-one (80\%), 1-(2-allyloxy-3,5-dichlorophenyl)-2-propen-1-one (73\%), 1-(2-allyloxy-5-bromophenyl)2-propen-1-one (71\%); $2 \mathrm{H}$-1-benzoxepin-5-one (2a) (81\%), 7,9-dichloro-2H-1-benzoxepin-5one (2b) (84\%), and 7-bromo-2H-1-benzoxepin-5-one (2c) (82\%) were prepared by known procedures described in our previous report. ${ }^{9}$

General procedure for the preparation of compounds (3a-g). To a mixture of salicylaldehyde (1a-c) $(5 \mathrm{mmol})$ and 1-benzoxepin-5-one (2a-c) $(5 \mathrm{mmol})$ was added DABCO (1.23 g, $11 \mathrm{mmol})$ in a mixture of 1,4-dioxane $(20 \mathrm{~mL})$ and water $(40 \mathrm{~mL})$. The resulting mixture was stirred at reflux for 1 day. Then, the reaction mixture was extracted with dichloromethane ( $3 \times 50 \mathrm{~mL})$. The combined organic layer was washed with brine, and dried over anhydrous $\mathrm{MgSO}_{4}$. After filtration, the filtrate was concentrated in vacuo to give crude 3a-g, which was purified by column chromatography (ethyl acetate: $n$-hexane: $1: 20$ ).

5a,6-Dihydro-12H-chromeno[2,3-c][1]benzoxepin-12-one (3a). Compound 3a (0.90 g, 68\%) was obtained as yellow liquid, $\mathrm{R}_{\mathrm{f}}=0.54$ (ethyl acetate: $n$-hexane $\left.=1: 6\right) ; \mathrm{IR}(\mathrm{KBr}) \mathrm{cm}^{-1}: 1643$ $(\mathrm{C}=\mathrm{O}) ;{ }^{1} \mathrm{H}-\mathrm{NMR}\left(\mathrm{CDCl}_{3}, 400 \mathrm{MHz}\right) \delta 4.39$ (dd, $\left.J 12.8,2.8 \mathrm{~Hz}, 1 \mathrm{H}, \mathrm{H}-6 \mathrm{a}\right), 4.79$ (dd, $J 12.8,4.0$ Hz, 1H, H-6b), 5.14 (ddd, $J$ 4.0, 2.8, 2.0 Hz, 1H, H-5a), 7.21 (m, 5H, ArH), 7.37 (d, J 2.0 Hz, $1 \mathrm{H}, \mathrm{H}-13$ ), 7.48 (td, $J 8.0,1.8 \mathrm{~Hz}, 1 \mathrm{H}, \mathrm{ArH}), 8.13$ (dd, $J$ 8.4, $1.8 \mathrm{~Hz}, 1 \mathrm{H}, \mathrm{ArH}$ ); ${ }^{13} \mathrm{C}-\mathrm{NMR}$ $\left(\mathrm{CDCl}_{3}, 100 \mathrm{MHz}\right) \delta 73.9,75.3,116.2,121.2,121.4,122.3,123.2,126.8,129.3,131.3,131.8$, 132.8, 133.7, 134.6, 154.5, 161.2, 180.2; EI-MS (70eV) $\mathrm{m} / \mathrm{z}$ (rel. intensity, \%) $264\left(\mathrm{M}^{+}, 55\right), 205$ 
(15), 176 (11), 146 (37), 144 (100), 117 (24), 115 (19); HRMS calcd for $\mathrm{C}_{17} \mathrm{H}_{12} \mathrm{O}_{3}$ : 264.0786. Found: 264.0788 .

2-Bromo-5a,6-dihydro-12H-chromeno[2,3-c][1]benzoxepin-12-one (3b). Compound $3 b$ (1.23 $\mathrm{g}, 72 \%$ ) was obtained as yellow crystals, $\mathrm{mp}=155-156{ }^{\circ} \mathrm{C} ; \mathrm{R}_{\mathrm{f}}=0.55$ (ethyl acetate: $n$-hexane $=$ 1: 6); IR (KBr) cm ${ }^{-1}: 1644(\mathrm{C}=\mathrm{O}) ;{ }^{1} \mathrm{H}-\mathrm{NMR}\left(\mathrm{CDCl}_{3}, 400 \mathrm{MHz}\right) \delta 4.39$ (dd, $J 12.8,2.8 \mathrm{~Hz}, 1 \mathrm{H}$, H-6a), 4.79 (dd, J 12.8, 4.0 Hz, 1H, H-6b), 5.13 (ddd, J 4.0, 2.8, 2.0 Hz, 1H, H-5a), 6.97 (d, $J 8.4$ $\mathrm{Hz}, 1 \mathrm{H}, \mathrm{ArH}), 7.01(\mathrm{~d}, J 8.4 \mathrm{~Hz}, 2 \mathrm{H}, \mathrm{ArH}), 7.30$ (dd, J 8.0, 1.6 Hz, 2H, ArH), 7.40 (d, J 2.0 Hz, $1 \mathrm{H}, \mathrm{H}-13), 8.25$ (d, J $2.8 \mathrm{~Hz}, 1 \mathrm{H}, \mathrm{ArH}) ;{ }^{13} \mathrm{C}-\mathrm{NMR}\left(\mathrm{CDCl}_{3}, 100 \mathrm{MHz}\right) \delta 74.1,75.1,116.1,116.3$, $121.3,122.5,123.2,127.9,129.5,132.2,133.0,133.7,133.8,137.3,154.6,160.3,186.3$; EI-MS (70eV) m/z (rel. intensity, \%) $341\left(\mathrm{M}^{+}, 1\right), 205$ (10), 176 (12), 145 (13), 144 (100), 116 (11), 115 (11); HRMS calcd for $\mathrm{C}_{17} \mathrm{H}_{11} \mathrm{BrO}_{3}$ : 341.9892. Found: 341.9889; Anal. Calcd for $\mathrm{C}_{17} \mathrm{H}_{11} \mathrm{BrO}_{3}$ : C, 59.50; H, 3.23. Found: C, 59.32; H, 3.51.

10-Bromo-5a,6-dihydro-12H-chromeno[2,3-c][1] benzoxepin-12-one (3c). Compound 3c (1.24 $\mathrm{g}, 73 \%$ ) was obtained as colorless liquid, $\mathrm{R}_{\mathrm{f}}=0.56$ (ethyl acetate $: n$-hexane $\left.=1: 6\right) ; \mathrm{IR}(\mathrm{KBr}) \mathrm{cm}^{-}$ 1: $1639(\mathrm{C}=\mathrm{O}) ;{ }^{1} \mathrm{H}-\mathrm{NMR}\left(\mathrm{CDCl}_{3}, 400 \mathrm{MHz}\right) \delta 4.38(\mathrm{dd}, J$ 12.8, $2.8 \mathrm{~Hz}, 1 \mathrm{H}, \mathrm{H}-6 \mathrm{a}), 4.79$ (dd, $J$ 12.8, 4.0 Hz, 1H, H-6b), 5.14 (ddd, J 4.0, 2.8, $2.0 \mathrm{~Hz}, 1 \mathrm{H}, \mathrm{H}-5 \mathrm{a}), 6.86$ (d, J 8.8 Hz, 1H, ArH), $7.13(\mathrm{td}, J$ 8.4, $1.2 \mathrm{~Hz}, 1 \mathrm{H}, \mathrm{ArH}), 7.17$ (td, $J$ 8.4, $1.2 \mathrm{~Hz}, 1 \mathrm{H}, \mathrm{ArH}), 7.36$ (m, 2H, ArH), 7.47 (d, $J$ $2.0 \mathrm{~Hz}, 1 \mathrm{H}, \mathrm{H}-13), 7.50$ (dd, $J 8.4,2.0 \mathrm{~Hz}, 1 \mathrm{H}, \mathrm{ArH}), 8.12(\mathrm{dd}, J 8.4,2.0 \mathrm{~Hz}, 1 \mathrm{H}, \mathrm{ArH}) ;{ }^{13} \mathrm{C}-$ NMR $\left(\mathrm{CDCl}_{3}, 100 \mathrm{MHz}\right) \delta 73.8,75.6,114.4,118.0,121.2,123.2,123.4,126.4,128.5,129.4$, 131.3, 131.4, 134.3, 134.9, 153.4, 161.4, 187.7; EI-MS (70eV) $\mathrm{m} / z$ (rel. intensity, \%) $341\left(\mathrm{M}^{+}\right.$, 20) 224 (98), 222 (100), 176 (17), 116 (16), 115 (43), 89 (14); HRMS calcd for $\mathrm{C}_{17} \mathrm{H}_{11} \mathrm{BrO}_{3}$ : 341.9892. Found: 341.9895 .

2,10-Dibromo-5a,6-dihydro-12H-chromeno[2,3-c][1]benzoxepin-12-one (3d). Compound 3d $(1.57 \mathrm{~g}, 75 \%)$ was obtained as colorless liquid, $\mathrm{R}_{\mathrm{f}}=0.53$ (ethyl acetate $: n$-hexane $=1: 6$ ); IR $(\mathrm{KBr}) \mathrm{cm}^{-1}: 1649(\mathrm{C}=\mathrm{O}) ;{ }^{1} \mathrm{H}-\mathrm{NMR}\left(\mathrm{CDCl}_{3}, 400 \mathrm{MHz}\right) \delta 4.37$ (dd, J 13.2, $\left.2.4 \mathrm{~Hz}, 1 \mathrm{H}, \mathrm{H}-6 \mathrm{a}\right), 4.79$ (dd, J 13.2, 4.0 Hz, 1H, H-6b), 5.12 (ddd, J 4.0, 2.4, $2.0 \mathrm{~Hz}, 1 \mathrm{H}, \mathrm{H}-5 \mathrm{a}$ ), 6.86 (d, J 8.8 Hz, 1H, ArH), 7.02 (d, J 8.8 Hz, 1H, ArH), 7.28 (d, J=2.0 Hz, 1H, H-13), 7.36 (dd, J 9.2, $2.4 \mathrm{~Hz}, 2 \mathrm{H}$, ArH), 7.56 (dd, $J$ 8.8, $2.8 \mathrm{~Hz}, 1 \mathrm{H}, \mathrm{ArH}), 8.24$ (d, $J 2.8 \mathrm{~Hz}, 1 \mathrm{H}, \mathrm{ArH}) ;{ }^{13} \mathrm{C}-\mathrm{NMR}\left(\mathrm{CDCl}_{3}, 100\right.$ MHz) $874.0,75.4,114.6,116.1,118.1,123.0,123.2,127.7,131.5,132.3,134.7,134.1,134.6$, 137.6, 153.5, 160.5, 186.2; EI-MS (70eV) m/z (rel. intensity, \%) $420\left(\mathrm{M}^{+}, 5\right), 225$ (12), 224 (99), 223 (14), 222 (100), 176 (12), 115 (26); HRMS calcd for $\mathrm{C}_{17} \mathrm{H}_{10} \mathrm{Br}_{2} \mathrm{O}_{3}$ : 419.8997. Found: 419.8993.

2-Bromo-8,10-dichloro-5a,6-dihydro-12H-chromeno[2,3-c][1]benzoxepin-12-one

(3e).

Compound $3 \mathrm{e}(1.51 \mathrm{~g}, 74 \%)$ was obtained as colorless liquid, $\mathrm{R}_{\mathrm{f}}=0.55$ (ethyl acetate : $n$-hexane = 1: 6); IR (KBr)cm ${ }^{-1}: 1644(\mathrm{C}=\mathrm{O})$; ${ }^{1} \mathrm{H}-\mathrm{NMR}\left(\mathrm{CDCl}_{3}, 400 \mathrm{MHz}\right) \delta 4.42$ (dd, $J 12.4,3.2 \mathrm{~Hz}, 1 \mathrm{H}$, H-6a), 4.85 (dd, J 12.4, 4.8 Hz, 1H, H-6b), 5.18 (ddd, J 4.8, 3.2, 1.6 Hz, 1H, H-5a), 6.96 (d, J 8.4 $\mathrm{Hz}, 1 \mathrm{H}, \mathrm{ArH}), 7.02$ (dd, J 8.8, 1.2 Hz, 1H, ArH), 7.30 (m, 1H, ArH), 7.46 (d, J 1.6 Hz, 1H, H13), $7.58(\mathrm{~d}, J 2.4 \mathrm{~Hz}, 1 \mathrm{H}, \mathrm{ArH}), 7.97(\mathrm{~d}, J 2.8 \mathrm{~Hz}, 1 \mathrm{H}, \mathrm{ArH}) ;{ }^{13} \mathrm{C}-\mathrm{NMR}\left(\mathrm{CDCl}_{3}, 100 \mathrm{MHz}\right) \delta$ 74.1, 74.7, 114.6, 118.2, 119.0, 122.6, 127.7, 129.0, 129.3, 131.7, 132.7, 133.3, 134.6, 134.9, 
153.2, 155.1, 185.6; EI-MS (70eV) m/z (rel. intensity, \%) $410\left(\mathrm{M}^{+}, 6\right), 225$ (13), 224 (100), 223 (17), 223 (100), 222 (97), 115 (26); HRMS calcd for $\mathrm{C}_{17} \mathrm{H}_{9} \mathrm{BrCl}_{2} \mathrm{O}_{3}$ : 409.9112 . Found: 409.9113. 2-Methoxy-5a,6-dihydro-12H-chromeno[2,3-c][1]benzoxepin-12-one (3f). Compound 3f $(1.13 \mathrm{~g}, 77 \%)$ was obtained as colorless liquid, $\mathrm{R}_{\mathrm{f}}=0.56$ (ethyl acetate $: n$-hexane $=1: 6$ ); IR $(\mathrm{KBr}) \mathrm{cm}^{-1}: 1646(\mathrm{C}=\mathrm{O}) ;{ }^{1} \mathrm{H}-\mathrm{NMR}\left(\mathrm{CDCl}_{3}, 400 \mathrm{MHz}\right) \delta 3.79\left(\mathrm{~s}, 3 \mathrm{H}, \mathrm{ArOCH}_{3}\right), 4.38(\mathrm{dd}, J$ 12.4, $2.8 \mathrm{~Hz}, 1 \mathrm{H}, \mathrm{H}-6 \mathrm{a}), 4.79$ (dd, J 12.4, 4.0 Hz, 1H, H-6b), 5.08 (ddd, J 4.0, 2.8, $2.0 \mathrm{~Hz}, 1 \mathrm{H}, \mathrm{H}-5 \mathrm{a}$ ), $6.78(\mathrm{~d}, J 2.8 \mathrm{~Hz}, 1 \mathrm{H}, \mathrm{ArH}), 6.85(\mathrm{dd}, J 8.8,3.2 \mathrm{~Hz}, 1 \mathrm{H}, \mathrm{ArH}), 6.91$ (d, $J 8.8 \mathrm{~Hz}, 1 \mathrm{H}, \mathrm{ArH}), 7.13$ (dd, J 8.4, 1.6 Hz, 1H, ArH), 7.17 (dd, J 8.0, 2.0 Hz, 1H, ArH), 7.34 (d, J 2.0 Hz, 1H, H-13), $7.48(\mathrm{td}, J$ 8.4, $2.0 \mathrm{~Hz}, 1 \mathrm{H}, \mathrm{ArH}), 8.14(\mathrm{dd}, J$ 8.0, $1.6 \mathrm{~Hz}, 1 \mathrm{H}, \mathrm{ArH}) ;{ }^{13} \mathrm{C}-\mathrm{NMR}\left(\mathrm{CDCl}_{3}, 100\right.$ $\mathrm{MHz}) \delta 55.8,73.9,75.5,113.0,117.0,118.1,121.2,121.9,123.2,126.6,131.4,133.0,134.5$, 134.7, 148.6, 154.7, 161.4, 187.8; EI-MS (70eV) $\mathrm{m} / \mathrm{z}$ (rel. intensity, \%) $294\left(\mathrm{M}^{+}, 25\right), 175$ (12), 174 (100), 159 (8), 146 (6), 131 (6), 103 (6); HRMS calcd for $\mathrm{C}_{18} \mathrm{H}_{14} \mathrm{O}_{4}$ : 294.0892. Found: 294.0893.

8,9-Dichloro-2-methoxy-5a,6-dihydro-12H-chromeno[2,3-c][1]benzoxepin-12-one

(3g). Compound $3 \mathrm{~g}$ (1.38 g, 76\%) was obtained as yellow crystals, $\mathrm{mp}=142-143{ }^{\circ} \mathrm{C} ; \mathrm{R}_{\mathrm{f}}=0.55$ (ethyl acetate: $n$-hexane $=1: 6)$; IR $(\mathrm{KBr}) \mathrm{cm}^{-1}: 1640(\mathrm{C}=\mathrm{O}) ;{ }^{1} \mathrm{H}-\mathrm{NMR}\left(\mathrm{CDCl}_{3}, 400 \mathrm{MHz}\right) \delta 3.79(\mathrm{~s}, 3 \mathrm{H}$, $\left.\mathrm{ArOCH}_{3}\right), 4.40$ (dd, J 12.4, $\left.3.2 \mathrm{~Hz}, 1 \mathrm{H}, \mathrm{H}-6 \mathrm{a}\right), 4.85$ (dd, $J$ 12.4, $4.8 \mathrm{~Hz}, 1 \mathrm{H}, \mathrm{H}-6 \mathrm{~b}$ ), 5.11 (ddd, $J$ 4.8, 3.2, 1.6 Hz, 1H, H-5a), 6.79 (d, J 2.4 Hz, 1H, ArH), 6.88 (dd, J 8.8, $2.8 \mathrm{~Hz}, 1 \mathrm{H}, \mathrm{ArH}$ ), 6.91 (d, J $8.8 \mathrm{~Hz}, 1 \mathrm{H}, \operatorname{ArH}), 7.42$ (d, J 1.6 Hz, 1H, H-13), 7.58 (d, J 2.4 Hz, 1H, ArH), 7.98 (d, J 2.4 $\mathrm{Hz}, 1 \mathrm{H}, \mathrm{ArH}) ;{ }^{13} \mathrm{C}-\mathrm{NMR}\left(\mathrm{CDCl}_{3}, 100 \mathrm{MHz}\right) \delta 55.8,74.2,74.6,113.1,117.2,118.8,121.4$, $119.4,120.4,124.8,121.4,127.7,128.8,132.9,134.4,148.4,154.8,186.7$; EI-MS $(70 \mathrm{eV}) \mathrm{m} / z$ (rel. intensity, \%) $362\left(\mathrm{M}^{+}, 30\right), 333$ (9), 319 (7), 175 (8), 174 (100), 159 (7), 103 (6); HRMS calcd for $\mathrm{C}_{18} \mathrm{H}_{12} \mathrm{Cl}_{2} \mathrm{O}_{4}$ : 362.0113. Found: 362.0110; Anal. Calcd for $\mathrm{C}_{18} \mathrm{H}_{12} \mathrm{Cl}_{2} \mathrm{O}_{4}$ : C, 59.53; $\mathrm{H}$, 3.33. Found: C, 59.42; H, 3.31 .

\section{Acknowledgements}

We are grateful to National Science Council of Taiwan (NSC-99-2113-M-037-007) for financial support. We are also grateful to Miss Pei-Lin Chen at the Northern Precious Instrumental Center of NSC, Taiwan for measuring the X-ray data.

\section{References}

1. Nising, C. F.; Brase, S. Chem. Soc. Rev. 2012, 41, 988-999. Doi: 10.1039/c1cs15167c

2. (a) Lesch, B.; Brase, S. Angew. Chem. Int. Ed. 2004, 43, 115-118. Doi: 10.1002/anie.200352154 (b) Ohnemüller, U. K.; Nising, C. F.; Nieger, M.; Brase, S. Eur. J. Org. Chem. 2006, 1535-1546. Doi: 10.1002/ejoc.200500887 
3. (a) Nising, C. F.; Ohhemuller, U. K.; Friedrich, A.; Lesch, B.; Steiner, J.; Schnockel, H.; Nieger, M.; Brase, S. Chem. Eur. J. 2006, 12, 3647-3654. Doi: 10.1002/chem.200501485 (b) Nising, C. F.; Ohhemuller, U. K.; Brase, S. Angew. Chem. Int. Ed. 2006, 45, 307-309. Doi: 10.1002/anie.200502913

4. Li, H.; Wang, J.; E-Nunu, T.; Zu, L.; Jiang, W.; Wei, S.; Wang, W. Chem. Commun. 2007, 507-509. Doi: 10.1039/b611502k

5. Kotame, P.; Hong, B. H.; Liao, J. H. Tetrahedron Lett. 2009, 50, 704-707. Doi: 10.1016/j.tetlet.2008.11.106

6. Luo, S. P.; Li, Z. B.; Wang, L. P.; Guo, Y.; Xia, A. B.; Xu, D. Q. Org. Biomol. Chem. 2009, 7, 4539-4546. Doi: 10.1039/b910835a

7. Rios, R.; Sunden, H.; Ibrahem, I.; Zhao, G. L.; Cordova, A. Tetrahedron Lett. 2006, 47 , 8679-8682. Doi: 10.1016/j.tetlet.2006.10.028

8. (a) Chen, P. Y.; Chen, H. M.; Chiang, M. Y.; Wang, Y. F.; Li, S. R.; Wang, T. P.; Wang, E. C. Tetrahedron 2012, 68, 3030-3036. Doi: 10.1016/j.tet.2012.02.023 (b) Chen, P. Y.; Huang, K. S.; Tsai, C. C.; Wang, T. P.; Wang, E. C. Org. Lett. 2012, 14, 4930-4933. Doi: 10.1021/o1302256y (c) Wang, E. C. Tetrahedron 2011, 67, 4155-4160.Doi: 10.1016/j.tet.2011.04.070 (d) Tsai, J. C.; Li, S. R.; Chiang, M. Y.; Chen, L. Y.; Chen, P. Y.; Lo, Y. F.; Wang, C. H.; Lin, C. N.; Wang, E. C. J. Org. Chem. 2009, 74, 789-8801. Doi: 10.1021/jo9015634

9. Li, S. R.; Chen, H. M.; Chen, L. Y.; Tsai, J. C.; Chen, P. Y.; Hsu, S. C.-N.; Wang, E. C. Arkivoc 2008, (ii), 172-182. Doi: 10.3998/ark.5550190.0009.219

10. The CIF file of crystal 3d has been deposited at the Cambridge Crystallographic Centre (CCDC), UK, and received the CCDC no. 862431. The X-ray crystallographic study of $\mathbf{3 d}$ is summarized as follows. Crystal data: $\mathrm{C}_{17} \mathrm{H}_{10} \mathrm{Br}_{2} \mathrm{O}_{3}, \mathrm{M}=422.07$, monoclinic system, space group P 1 21/c 1, $a=4.28210(10) \AA, b=13.1164(3) \AA, c=26.6831(6) \AA, V=1498.65(6) \AA^{3}$, $Z=4, \mathrm{~d}=1.871 \mathrm{Mg} / \mathrm{m}^{3}$. A crystal of dimensions $0.23 \times 0.02 \times 0.02 \mathrm{~mm}^{3}$ was used for measurements on a RIGAKU AFC7S diffractometer with a graphite monochromator $(\omega$ scans, $\left.2 \theta_{\max }=45.0^{\circ}\right)$, Mo K $\alpha$ radiation $(\lambda=0.71073 \AA)$. The total number of independent reflections measured was 3066 [R(int) $=0.0386$ ], of which 23771 were observed. The crystal structure was solved by the direct method and expanded using difference Fourier techniques, further refined by the program SHELXTL 97 and full-matrix least-squares calculations. Final indices: $\mathrm{R}_{\mathrm{f}}=0.0701, \mathrm{R}_{\mathrm{w}}=0.0819\left(\mathrm{w}=1 /\left[\sigma^{2}\left(\mathrm{~F}_{\mathrm{o}}^{2}\right)+(0.1692 \mathrm{P})^{2}\right]\right.$ where $\left.\mathrm{P}=\left(\mathrm{F}_{\mathrm{o}}^{2}+2 \mathrm{~F}_{\mathrm{c}}^{2}\right) / 3\right)$. The $\mathrm{CIF}$ file is also available in supplementary data. [Please note the carbon numbering in ORTEP is different from that of carbon numbering in its chemical nomenclature]. 